\title{
S-Matrix Oriented CAD-Tool for Simulating Complex Integrated Optical Circuits
}

\author{
X. J. M. Leijtens, P. Le Lourec, and M. K. Smit, Associate Member, IEEE
}

\begin{abstract}
A design tool for simulation of complex integrated optical circuits, based on a professional microwave design system has been developed. Implementation of a number of components is described and a simulation example of an add-drop node using a $4 \times 4$ phased array is presented.
\end{abstract}

\section{INTRODUCTION}

C OMPUTER-AIDED design (CAD) tools for integrated optical circuits are far less developed than their microwave counterparts. In general commercially available tools initiate the design and simulation based on a layout description of the circuit and use BPM based computational techniques to carry out the simulation.

Computer-aided design tools for microwave integrated circuits use a more abstract approach initiating the design on a symbolic level. From this level the simulation is performed and the layout is generated (see Fig. 1). The advantage of this approach is that the circuit is better structured and that both BPM and other simulation methods can be implemented for different components or sub circuits. Observing that integrated optical circuits are conceptually quite similar to microwave integrated circuits, we have chosen to adapt a professional microwave design system, Hewlett Packard's MDS, for the CAD of optical chips [1], rather than developing a system completely from scratch. The standard tool provides a graphical interface for building circuits with standard or user-defined components and it performs (multi-) parameter sweeps and automatic (multi-) parameter optimizations. Simulation results are processed and presented on screen, or exported to other applications. Mask layout can be (automatically) generated, manipulated and viewed on the screen. Subcircuits are grouped into new circuit components facilitating hierarchical designs. All these facilities are applicable to optical designs without adaptation. The main effort in changing the tool into an optical design tool has been the implementation of a number of components into the simulator, for handling propagation and coupling of optical fields.

\section{DESCRIPTION OF OPTICAL COMPONENTS}

The coupling between optical components takes place via guided modes and radiation fields. If coupling through radi-

Manuscript received July 23, 1996; revised October 10, 1996. This work was supported by CNET LAB/FCI/PIH. Part of this work has been carried out in the ACTS-BLISS AC-065 project.

X. Leijtens and M. Smit are with Delft University of Technology, P.O. Box 5031, 2600 GA Delft, The Netherlands.

P. Le Lourec is with France Telecom, CNET, Technopole Anticipa, 22307 Lannion, France.

Publisher Item Identifier S 1077-260X(96)09505-6.

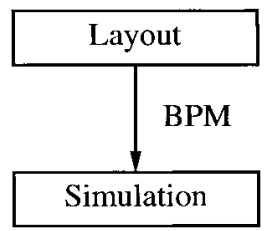

(a)

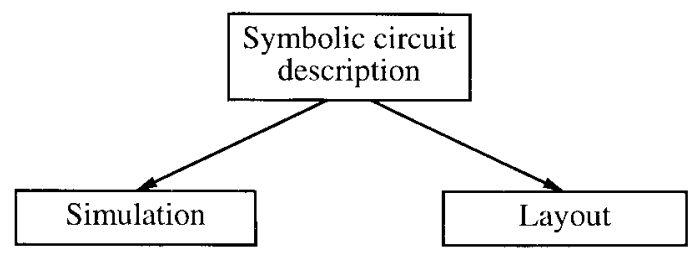

(b)

Fig. 1. BPM simulation from physical description (a). Simulation and layout generation from a symbolic description (b).
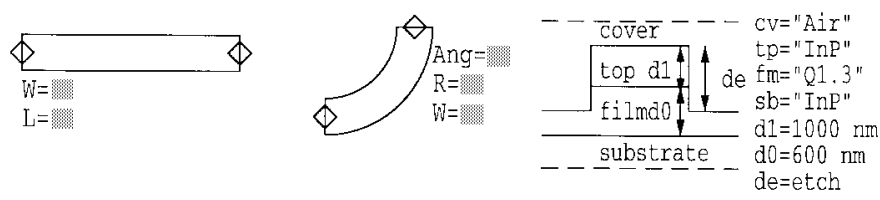

Fig. 2. Symbolic representation of a straight waveguide, a curved waveguide and of a layer structure.

ation fields is small, optical components can be considered as individual units connected to each other at well defined ports. In this concept, the response of an $N$-port component is described by its $N \times N S$-matrix [2]. For components where coupling through radiation fields plays an important role, the part where this coupling takes place can be included in the component simulation using a beam propagation method (BPM) or a diffraction routine for computing the propagation through this component. This has been done for example with the simulation model of the phased array, as described below.

An ideal monomode waveguide is a two-port component. The propagation in a straight waveguide is described by $S_{12}=S_{21}=e^{-j \beta \ell}$, with $\beta$ the propagation constant and $\ell$ the length of the waveguide. For a curved waveguide $S_{12}=$ $S_{21}=e^{-j \beta_{\phi} \phi}$, with $\beta_{\phi}$ the angular propagation constant and $\phi$ the angle of the curved waveguide. Since there are no reflections in the waveguide, $S_{11}=S_{22}=0$. The symbolic representation of a monomode straight and curved waveguide are shown in Fig. 2(a) and (b). Each optical component is connected to a symbol specifying the waveguide structure. An example representative of the layer structure of a strip-loaded 


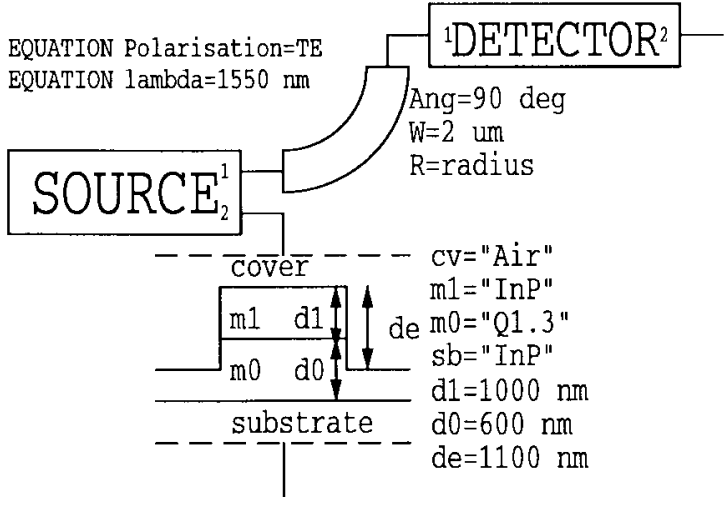

(a)

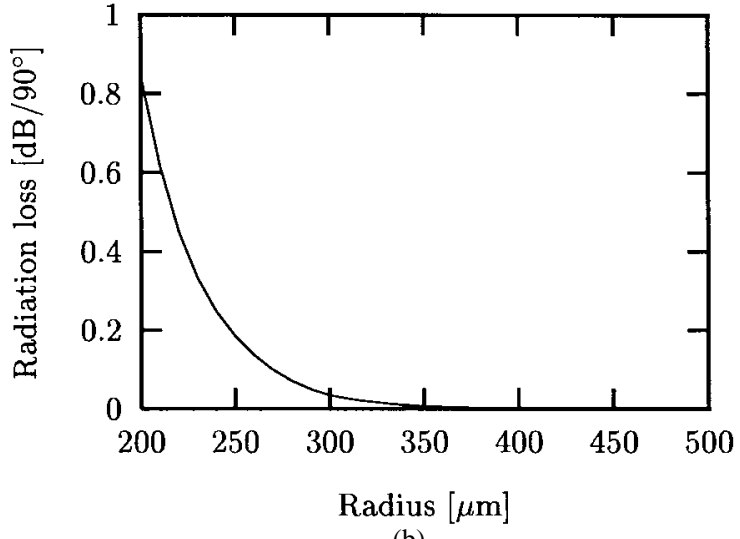

(b)

Fig. 3. (a) Symbolic representation of an optical circuit consisting of a single $90^{\circ}$ circularly curved waveguide and (b) the result of a simulation of the radiation loss as a function of the radius of curvature.

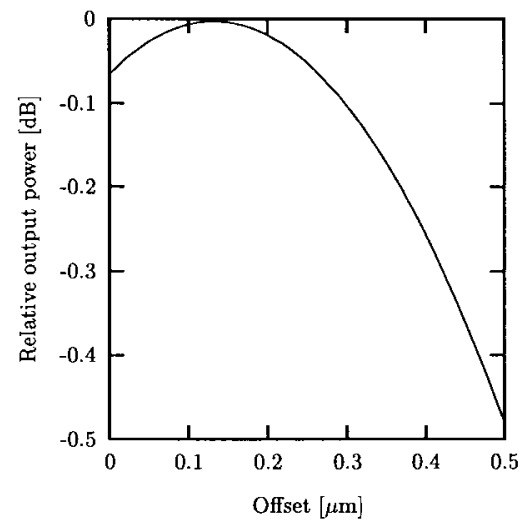

(a)

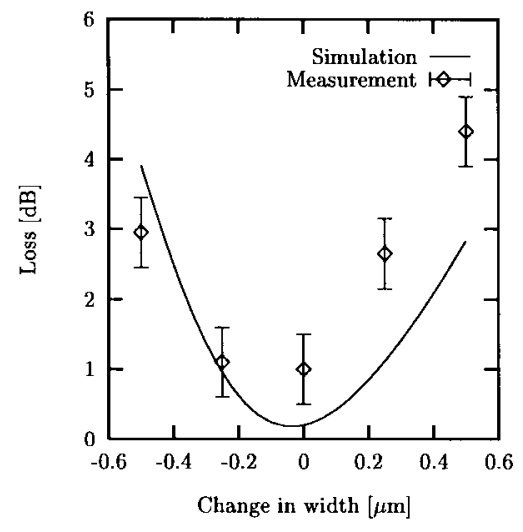

(b)

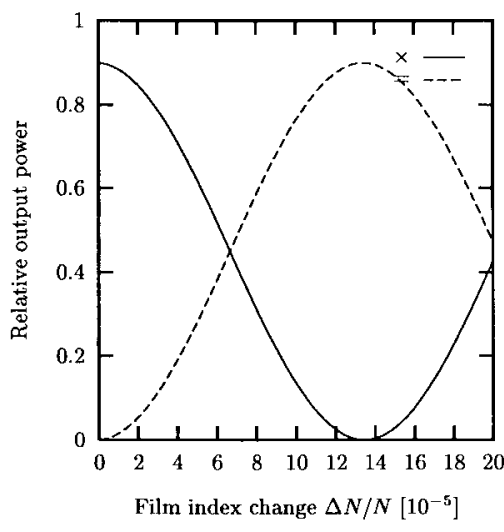

(c)

Fig. 4. (a) Simulation of the coupling loss at a straight-curved junction as a function of the offset. (b) Simulation of relative output power in the cross output of an MZI switch with 16- $\mu \mathrm{m}$-wide 3-dB MMI couplers, as a function of a change in the width of all waveguide structures. The simulations have been performed with the EIM and are compared to measurements on realized devices. Each point is the average of two measurements. (c) Relative output power in the cross $(x)$ and bar $(=)$ outputs for an MZI switch, as a function of a change in the film index in one MZI arm.

rib waveguide is shown in Fig. 2(c). In order to obtain the $S$ matrix, any mode solver calculating the modal propagation constants can be used. The current implementation offers the choice of the effective index method (EIM), in combination with a conformal transformation for curved waveguides [3], and a two-dimensional (2-D) finite element method (FEM). As an example the result of a simulation of the radiation loss in a curved waveguide, as a function of the radius of curvature is shown in Fig. 3, together with the symbolic representation of the circuit ${ }^{1}$.

The junction between two monomode waveguides is described by a $2 \times 2$ scattering matrix. The matrix elements are given by the overlap integral of the modal fields, $U_{1}$ and $U_{2}$, in each of the waveguides: $S_{12}^{*}=S_{21}=\int U_{1} U_{2}^{*}$. As reflections in optical chips are small for many applications, $S_{11}$ and $S_{22}$ are put to zero, although inclusion of reflections is straightforward. The scattering matrices for multimode waveguides and junctions are determined based on calculations of the modal propagation constants $\beta_{\nu}$ and the corresponding modal field distributions $U_{\nu}$. One port is used for each mode

\footnotetext{
${ }^{1}$ All simulations in this article have been performed for TE polarization.
}

in the waveguide or junction. Fig. 4(a) shows a simulation result for optimising the offset at a straight-curved waveguide junction. This simulation was performed for a junction between a $2-\mu \mathrm{m}$-wide straight waveguide and a $2-\mu \mathrm{m}$-wide curved waveguide with a radius of $500 \mu \mathrm{m}$ with a waveguide structure as shown in Fig. 3. The optimum offset as read from the figure is $0.13 \mu \mathrm{m}$.

Components are combined into circuits as shown in Fig. 5, where curved access waveguides are connected by a $1 \times$ 2 junction with a multimode straight waveguide to form an MMI coupler. Two MMI couplers are connected with straight waveguides to create an optical circuit representing a Mach-Zehnder interferometer (MZI) switch [4]. The full MZI circuit can be represented by a single symbol. The performance of the MZI switch, after optimization of each of the composing components, as a function of changes in the waveguide and MMI widths has been simulated and compared to measurements on realized switches (see Fig. 4(b)). The MMI couplers used in this switch measure $16 \times 400 \mu \mathrm{m}^{2}$ (nominal value). The shape of the simulated curve agrees well with the measurements. The minima coincide within the 


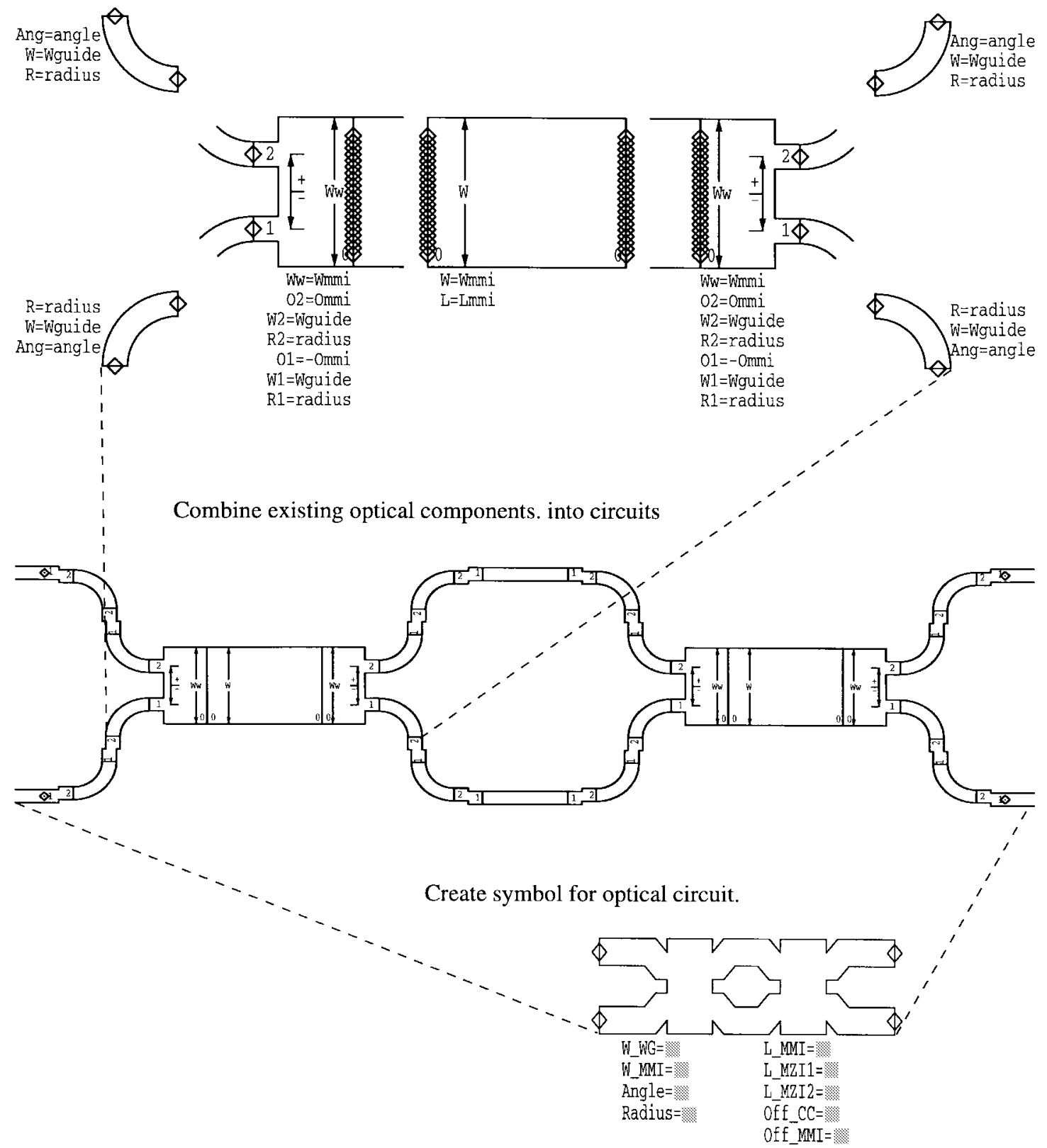

Fig. 5. Separate components are combined to form an optical circuit. The circuit is represented by a single symbol.

measurement error of $\pm 0.1 \mu \mathrm{m}$. The measurements have a higher loss than the simulation. The simulated response of the MZI as a function of the electro-optic change in film index in one of the interferometer arms is presented in Fig. 4(c).

A $4 \times 4$ phased array has been implemented in MDS. The symbol is shown in Fig. 6(a). The simulation is performed in two steps. First the geometry of the phased array is created with the desired specifications, such as the number of input and output ports, the central wavelength and the channel spacing. Secondly, the propagation through this phased array is simulated.

The design of the geometry of the phased array is based on the description by Smit [5] and uses a combination of two star couplers connected by an array of straight and curved waveguides. A phased array with $N$ input and $M$ output waveguides is described by an $(N+M) \times(N+M) S$-matrix.
The matrix elements are given by

$$
S_{i j}= \begin{cases}0, & \text { if } i, j \leq N \text { or } i, j>N \\ C_{i j} & \text { otherwise }\end{cases}
$$

with $i, j \in[1, \cdots, N+M]$, where $C_{i j}$ is the transmission coefficient from port $i$ to port $j$. These coefficients are calculated in the following way: first the field from port $i$ is diffracted and the coupling with each arm $k$ of the waveguide array in the phased array is calculated. This yields a coupling matrix $A_{i k}$, if monomode array arms are assumed. Secondly, the propagation in each of the waveguide arms is calculated. For each array arm, it takes into account the coupling loss at the junctions and the radiation loss in the curved waveguides. This gives a vector $B_{k}$. Thirdly the coupling matrix from the phased array arms to the output port $j$ is calculated. This is in fact the complex conjugate of the input coupling matrix. 


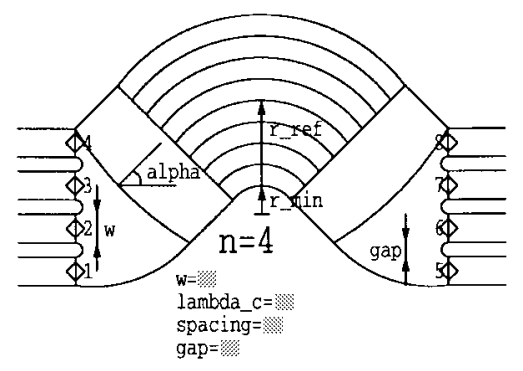

(a)

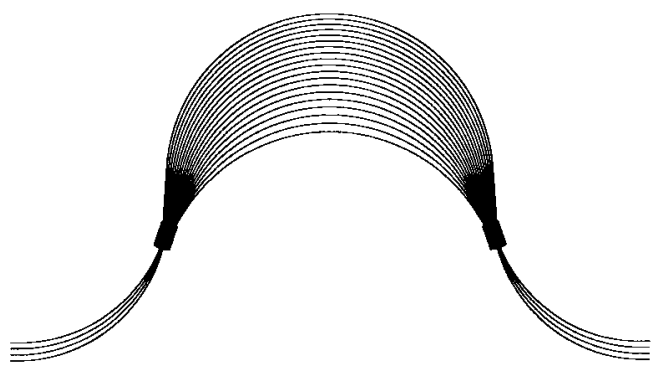

(b)

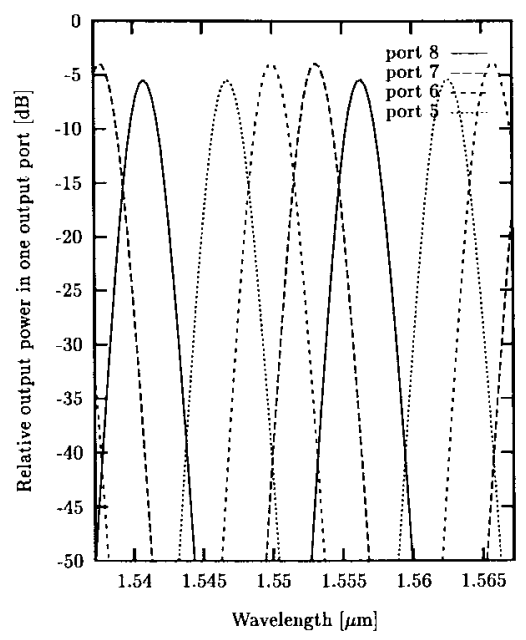

(c)

Fig. 6. Symbolic representation of (a) a $4 \times 4$ phased array and (b) an example of the layout generated by this component. The wavelength response of the phased array for the add-drop application is shown in (c), when the signal is launched in input port 1 .

Thus, we obtain

$$
C_{i j}=\sum_{k=1}^{N_{a}} A_{i k} B_{k} A_{j k}^{*}
$$

with $N_{a}$ the number of arms in the waveguide array.

\section{Simulation OF AN ADD-Drop CiRCUIT}

The symbolic representation of a three channel add-drop node is shown in Fig. 7 and consists of one $4 \times 4$ phased array, three switches and interconnecting straight and curved waveguides. It operates as follows. Three signals with wavelengths $\lambda_{1}, \lambda_{2}$ and $\lambda_{3}$ are launched in the lower port (1) of the phased array. They are spatially separated by the phased array and exit at the upper three ports $(6,7$, and 8$)$ of the phased array. The separate wavelengths then go through a switch and, if each switch is in the cross state, go back through the phased array where the three signals are multiplexed into the lower output port (5).

If the signal $\lambda_{2}$ has to be dropped, switch 2 has to be put in the bar state. The signal at $\lambda_{2}$ exits at the port labeled "DROP 2 " and a signal of the same wavelength $\lambda_{2}$ added at the port labeled "ADD 2" is combined by the phased array with $\lambda_{1}$ and $\lambda_{3}$ and exits at the lower output port of the phased array.

A simulation of the add-drop has been performed for a circuit with the following specifications. The phased array was designed for a central wavelength of $1.5468 \mu \mathrm{m}$ and a channel spacing of $3.2 \mathrm{~nm}(400 \mathrm{GHz})$. This design gives $\lambda_{1}=1.5468$ $\mu \mathrm{m}, \lambda_{2}=1.5500 \mu \mathrm{m}, \lambda_{3}=1.5532 \mu \mathrm{m}$. A fourth channel at $\lambda_{4}=1.5564 \mu \mathrm{m}$ is included in the simulation for reference only. The input and output waveguides are $2-\mu \mathrm{m}$ wide and are $1.5 \mu \mathrm{m}$ apart. The phased array has 19 array arms each $2 \mu \mathrm{m}$ wide with a minimum separation of $0.5 \mu \mathrm{m}$. The layout of this phased array is shown in Fig. 6, together with the simulated spectral response at each of the output channels. Each MZI switch consists of two MMI couplers with a width of $16 \mu \mathrm{m}$ and a length of $400 \mu \mathrm{m}$. The MZI arms have a length of 2 $\mathrm{mm}$ and required a $0.0135 \%$ change of refractive index of the film layer in order to switch from cross to bar state [see Fig. 4(c)]. The length of the loops, including the phased array, is approximately $10.5,8.5$, and $6.5 \mathrm{~mm}$ for the outer, middle and inner loop, respectively. The complete circuit consists of 183 components (6 multimode and 32 monomode straight waveguides, 60 curved waveguides, 84 junctions and 1 phased array). The layer stack used is shown in Fig. 2(c), with an etch depth of $1.1 \mu \mathrm{m}$.

Two simulations have been performed varying the wavelength of the incoming signal. The first with all three switches in the cross state and the second with switch 2 in the bar state, thus dropping $\lambda_{2}$. The results of both simulations are shown in Fig. 8(a) and (b), respectively. Each simulation contains 350 points and takes four hours of CPU time on a (low end) HP712/80 workstation.

Fig. 8(a) shows four peaks at $\lambda_{1}, \cdots, \lambda_{4}$. Each signal at $\lambda_{1}, \lambda_{2}$ and $\lambda_{3}$ is routed by the phased array to its switch and loops back to the phased array. It thus suffers twice the loss of the phased array and once the loss of the switch. The signal at $\lambda_{4}$ is routed directly from input to output, and suffers the loss of the phased array only once. Even though the path length of the loop for $\lambda_{3}$ is the largest, the two peaks $\lambda_{1}$ and $\lambda_{3}$ are equally high, since the transmission loss in the waveguides is not taken into account. The peak at $\lambda_{1}$ is slightly lower because of the wavelength response of the phased array [see Fig. 6(c)]. Furthermore, since the phased array acts as a passband filter, the width of the peaks at $\lambda_{1}, \lambda_{2}$, and $\lambda_{3}$ is reduced since they are filtered twice. The modulation of the signal at $\lambda_{3}$ is due to the interference between the direct and the looped-back signal and has been observed in measurements of a similar add-drop circuit [6]. This interference phenomenon is strongly reduced for the other two channels, because the direct signal is greatly suppressed.

Fig. 8(b) shows the output at the detector (solid line) when switch 2 is in the bar state. The signal centred at $\lambda_{2}$ is dropped. The signal added at port "ADD 2" exits at the phased array 


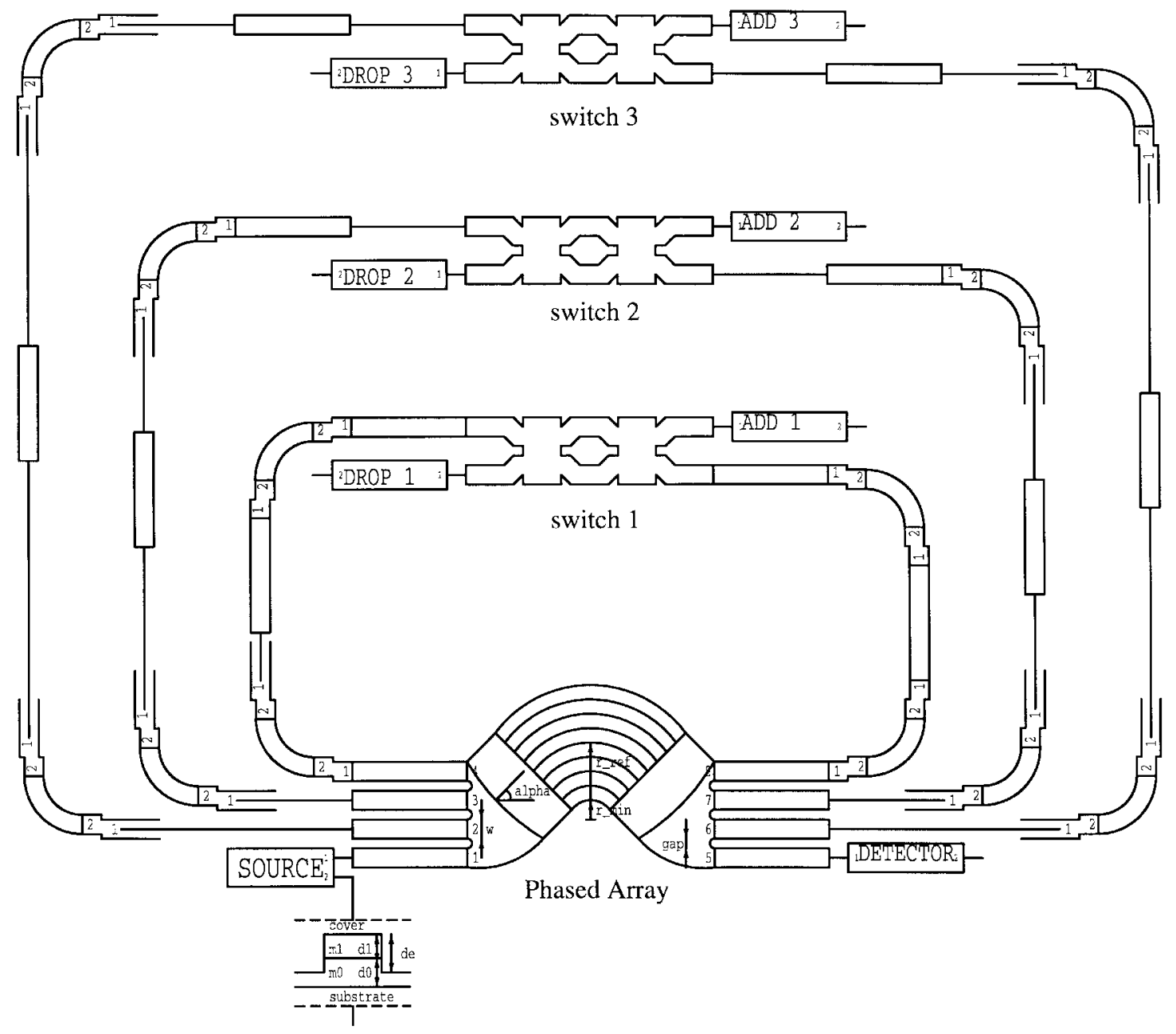

Fig. 7. Symbolic representation of the add-drop circuit.

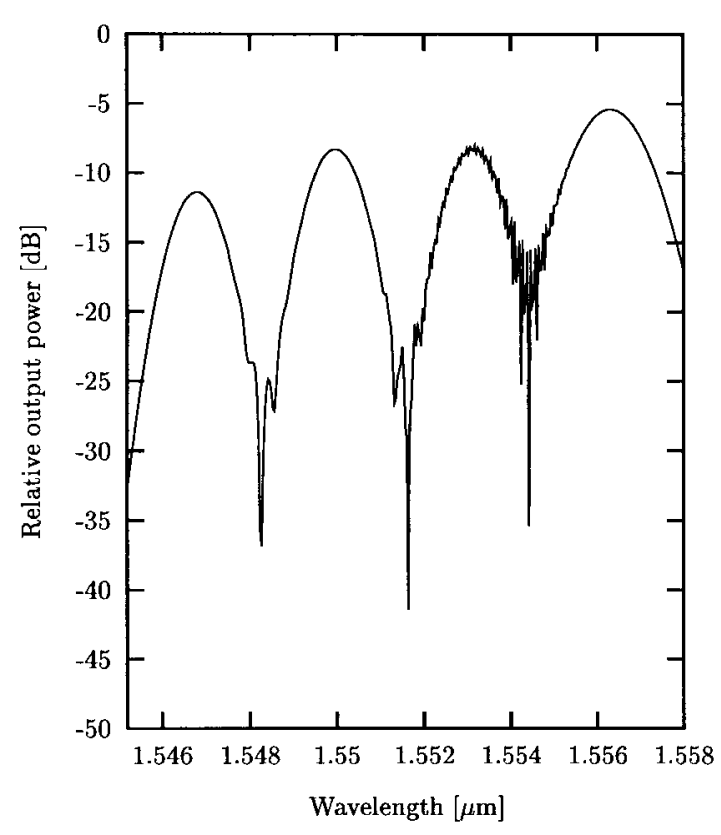

(a)

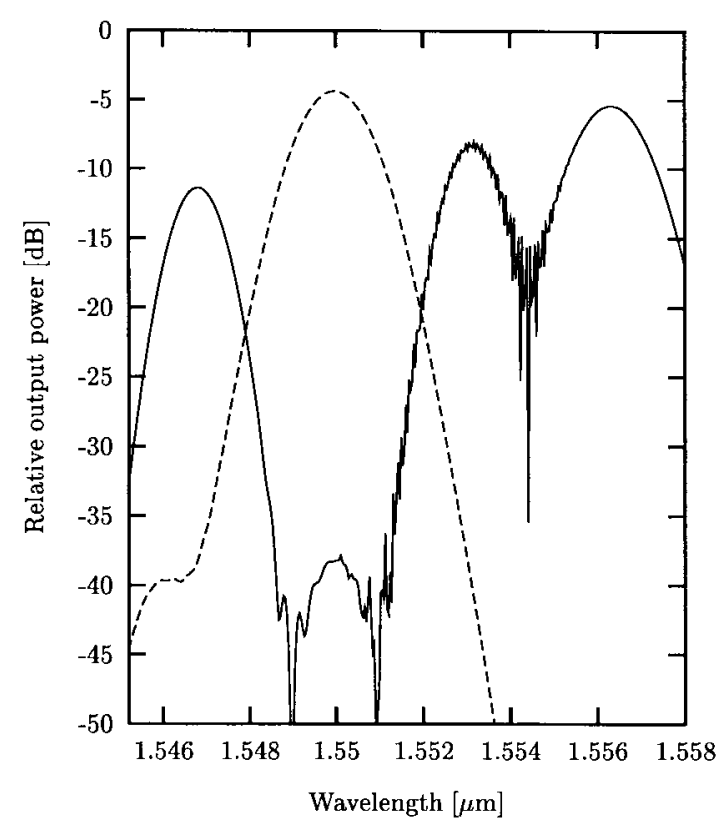

(b)

Fig. 8. Simulation results of a three channel add-drop node. (a) Response at the output of the add-drop. All switches are in the cross state. (b) Signal at the output of the add-drop when switch 2 is in the bar state. The added signal is shown with a dashed line.

output (dashed line). This peak is higher and wider than the In order to estimate the influence of the fabrication on the adjacent peaks, because it passes the phased array only once. device performance, the width of all waveguide structures in 


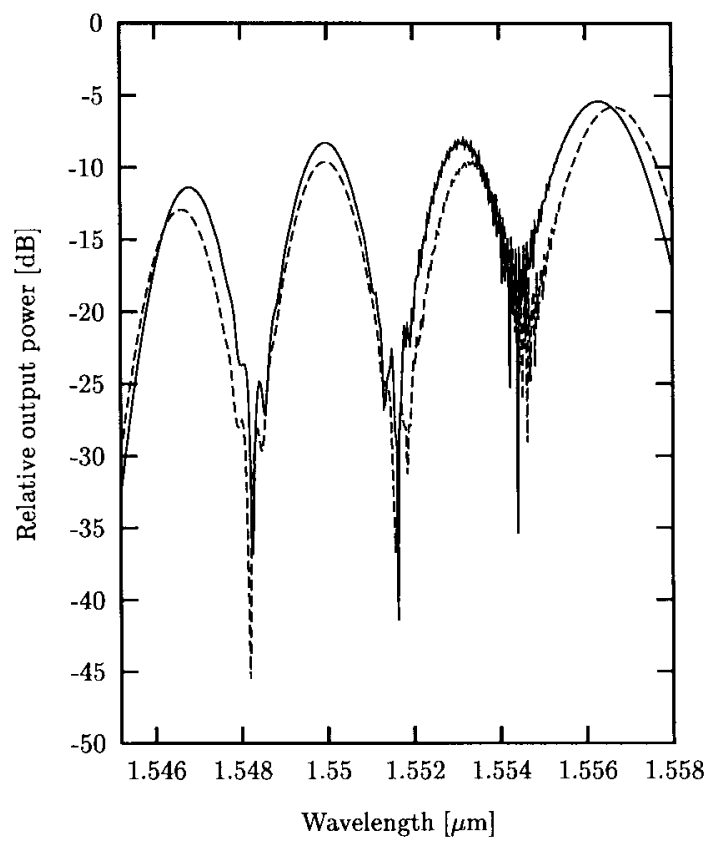

Fig. 9. Simulated response of the add-drop node if the width of all waveguide structures in the circuit is reduced by $0.2 \mu \mathrm{m}$ (dashed line). For comparison, the simulated response of the circuit for the nominal width has been included in the plot (solid line).

the circuit has been reduced by $0.2 \mu \mathrm{m}$. The simulation of this circuit is compared to that of the unmodified circuit. The result of the simulation is shown in Fig. 9. The variation of the width leads to a change in the phased array channel spacing from 3.2 to $3.4 \mathrm{~nm}$. From the peak at $\lambda_{4}$, one can deduce the extra insertion loss of the phased array. The signals at the other three peaks suffer twice this additional loss and that of the loop. An extra insertion loss of $0.4 \mathrm{~dB}$ for the phased array and $0.6 \mathrm{~dB}$ for the loop, including the switch, have been inferred from the simulated data.

\section{CONCLUSION}

An example of an application of a powerful CAD-tool for integrated optical circuits has been presented. The $S$-matrix approach enables the simulation of complex circuits, possibly containing loops, such as the one analyzed in this article. The simulation of the fabrication tolerance of a full circuit has been presented, which is of key importance for predicting the performance of a device.

\section{ACKNOWLEDGMENT}

The authors thank T. Uitterdijk for providing the experimental data for Fig. 4(b).

\section{REFERENCES}

[1] C. van Dam, L.C.N. de Vreede, M.K. Smit, J.L. Tauritz, and B.H. Verbeek, "Optical chip design with a microwave CAD-system," in Proc. 10th Eur. Conf. Circuit Theory and Design. New York: Polytechnic, Sept. 2-6, 1991, vol. III, pp. 1316-1323.

[2] D. Kerns and R. Beatty, Basic theory of Waveguide Junctions and Introductory Microwave Network Analysis. Oxford, U.K.: Pergamon, 1967.

[3] M. Heiblum and J. H. Harris, "Analysis of curved optical waveguides by conformal transformation," IEEE J. Quantum Electron., vol. QE-11, pp. 75-83, Feb. 1975; correction, vol. QE-12, p. 313, 1976.

[4] X. J. M. Leijtens, L. H. Spiekman, C. van Dam, L. C. N. de Vreede, M. K. Smit, and J. L. Tauritz, "CAD-tool for integrated optics," in Proc. 7th Conf. Integrated Optics (ECIO '95), Delft, The Netherlands, Apr. 3-6 1995, pp. 463-466.

[5] M. K. Smit, "Integrated optics in silicon-based aluminum oxide," Ph.D. thesis, Delft Univ. of Technol., Delft, The Netherlands, 1991.

[6] C. G. M. Vreeburg, T. Uitterdijk, Y. S. Oei, M. K. Smit, F. H. Groen, E. G. Metaal, P. Demeester, and H. J. Frankena, "First InP-based reconfigurable integrated add-drop multiplexer," IEEE Photon. Technol. Lett., vol. 9, pp. 188-190, Feb. 1997.

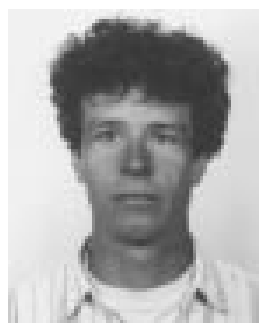

X. J. M. Leijtens was born in Uithoorn, the Netherlands, on April 6, 1962. He received the masters degree with honors in physics from the University of Amsterdam in 1988, the Ph.D. degree from the National Institute for Nuclear and High Energy Physics (NIKHEF-H) in Amsterdam in 1993 for research work at CERN in Geneva on the production of tau leptons from $\mathrm{Z}$ boson decay.

Since 1993, he is a Research Scientist at the Delft University of Technology. His current work is in the field of integrated optics and includes the design of multimode interference couplers, optical switches and wavelength duplexers. $\mathrm{He}$ is working on the development of a computer-aided design tool for integrated optical circuits.

P. Le Lourec, photograph and biography not available at the time of publication.

M. K. Smit (A'93), for photograph and biography, see this issue, p. 150. 